\title{
An international neutron cross section standards evaluation
}

\author{
Allan Carlson ${ }^{1, a}$, Sergey Badikov², Chen Zhenpeng ${ }^{3}$, Evgenij Gai ${ }^{2}$, Gerald Hale ${ }^{4}$, Franz-Josef Hambsch ${ }^{5}$, \\ Hartmut Hofmann ${ }^{6}$, Toshihiko Kawano ${ }^{4}$, Nancy Larson ${ }^{7}$, Soo-Youl Oh ${ }^{8}$, Vladimir Pronyaev ${ }^{2}$, Donald Smith ${ }^{9}$, \\ Siegfried Tagesen ${ }^{10}$, and Herbert Vonach ${ }^{10}$ \\ 1 National Institute of Standards \& Technology, 100 Bureau Dr. MS 8463, Gaithersburg, MD 20899-8463, USA \\ 2 Institute of Physics \& Power Engineering, Bondarenko sq. 1, 249033 Obninsk, Kaluga Region, Russia \\ 3 Tsinghua University, Beijing 100084, China \\ ${ }^{4}$ Los Alamos National Laboratory, T-2, MS B-243, Los Alamos, NM 87545, USA \\ 5 Institute for Reference Materials and Measurements, Retieseweg 111, 2440 Geel, Belgium \\ ${ }^{6}$ Erlangen-Nuernberg University, Staudtstrasse 7, 91058 Erlangen, Germany \\ 7 Oak Ridge National Laboratory, Bldg. 5700, MS 6171, P.O. Box 2008, Oak Ridge, TN 37831-6171, USA \\ 8 Korea Atomic Energy Research Institute, P.O. Box 105, Yuseong, Daejeon 305-600, Republic of Korea \\ 9 Argonne National Laboratory, 9700 S. Cass Avenue, Argonne, IL 60439, USA \\ 10 Vienna University, Waehringerstrasse 17, 1090 Vienna, Austria
}

\begin{abstract}
A new evaluation of the neutron cross section standards was recently completed. The results of this work became the standards for the new ENDF/B-VII library. This evaluation was performed to include new experiments on the standards that have been made since the ENDF/B-VI standards evaluation was completed and to improve the evaluation process. The evaluations include the $\mathrm{H}(\mathrm{n}, \mathrm{n}),{ }^{6} \mathrm{Li}(\mathrm{n}, \mathrm{t}),{ }^{10} \mathrm{~B}(\mathrm{n}, \alpha),{ }^{10} \mathrm{~B}\left(\mathrm{n}, \alpha_{1} \gamma\right),{ }^{197} \mathrm{Au}(\mathrm{n}, \gamma),{ }^{235} \mathrm{U}(\mathrm{n}, \mathrm{f})$, and ${ }^{238} \mathrm{U}(\mathrm{n}, \mathrm{f})$ standard reactions. Evaluations were not done for the ${ }^{3} \mathrm{He}(\mathrm{n}, \mathrm{p})$ and $\mathrm{C}(\mathrm{n}, \mathrm{n})$ standards which were carried over from ENDF/B-VI. The need for standards above $20 \mathrm{MeV}$ led to the extension of the ${ }^{235} \mathrm{U}(\mathrm{n}, \mathrm{f})$ and ${ }^{238} \mathrm{U}(\mathrm{n}, \mathrm{f})$ cross sections to $200 \mathrm{MeV}$. In addition evaluations were produced for the non-standard ${ }^{238} \mathrm{U}(\mathrm{n}, \gamma)$ and ${ }^{239} \mathrm{Pu}(\mathrm{n}, \mathrm{f})$ reactions. Results of this work and comparisons with previous evaluations are presented.
\end{abstract}

\section{Introduction}

Nuclear data calculations generally use comprehensive libraries of evaluated nuclear cross sections. Most of the cross sections that form the database for these evaluations have been measured relative to neutron cross section standards. Improvements in a standard allow all cross sections measured relative to that standard to be improved, thus emphasizing the need to improve the neutron cross section standards. Significant improvements have been made in the standard cross section database since the last complete evaluation of the neutron cross section standards 20 years ago. It is important to reevaluate these cross sections taking into account new experimental data and improved evaluation techniques. In response to requests for improvements in the standards, the Cross Section Evaluation Working Group (CSEWG) formed a Task Force, the Working Party on International Evaluation Cooperation (WPEC) of the Nuclear Energy Agency, Nuclear Science Committee formed a Subgroup, and the International Atomic Energy Agency (IAEA) formed a Coordinated Research Project (CRP). These groups have worked cooperatively to improve the evaluation process. The emphasis has been on the $\mathrm{H}(\mathrm{n}, \mathrm{n}),{ }^{10} \mathrm{~B}$ and fission standards. Table 1 shows the standards and the energies where they are considered standards. Extended energy ranges compared with the ENDF/B-VI results were obtained from the present evaluation for the cross sections for ${ }^{235} \mathrm{U}(\mathrm{n}, \mathrm{f})$ and ${ }^{238} \mathrm{U}(\mathrm{n}, \mathrm{f})$ from $20 \mathrm{MeV}$ to $200 \mathrm{MeV}$; and for ${ }^{10} \mathrm{~B}(\mathrm{n}, \alpha)$ and ${ }^{10} \mathrm{~B}\left(\mathrm{n}, \alpha_{1} \gamma\right)$ from $250 \mathrm{keV}$ to $1 \mathrm{MeV}$. Work is continuing on the extension

${ }^{a}$ Presenting author, e-mail: carlson@nist.gov of the $\mathrm{H}(\mathrm{n}, \mathrm{n})$ standard to $200 \mathrm{MeV}$. The ${ }^{238} \mathrm{U}(\mathrm{n}, \mathrm{f})$ cross section, which is an NEANDC/INDC standard, was accepted as an ENDF standard. However, $2 \mathrm{MeV}$ was recommended as the lower bound for use of this cross section as a standard. The use of this cross section from threshold to $2 \mathrm{MeV}$ is discouraged as a standard due to the very rapid change and the very small values of this cross section in that energy range. The present evaluation of this cross section does extend down to $1 \mathrm{MeV}$, where it can be used for some non-standard applications.

A very important contribution to the evaluation process was made by the IAEA CRP. The main objectives of the evaluation were the following: improve the methodology for determination of the covariance matrix used in cross section evaluations; upgrade the computer codes using this methodology; study the reasons for uncertainty reduction in R-matrix and model independent fits; evaluate cross sections and covariance matrices for the light elements, $\mathrm{H}(\mathrm{n}, \mathrm{n})$, ${ }^{3} \mathrm{He}(\mathrm{n}, \mathrm{p}),{ }^{6} \mathrm{Li}(\mathrm{n}, \mathrm{t}),{ }^{10} \mathrm{~B}\left(\mathrm{n}, \alpha_{1} \gamma\right)$, and ${ }^{10} \mathrm{~B}(\mathrm{n}, \alpha)$; establish the methodology and computer codes for combining the light element with the heavy element evaluations, leading to a final evaluation of the neutron cross section standards.

The evaluation work included improvements to the experimental data in the standards database and methods for handling discrepant data; R-matrix evaluation of the hydrogen scattering cross section and conversion of measurements relative to the hydrogen cross section to the new standard; studies of the effect of Peelle's Pertinent Puzzle (PPP) and its effect on the standards; evaluation work on microscopic calculations leading to independent determinations of R-matrix poles; studies of the small uncertainties resulting from evaluations; smoothing procedures; and finally, results provided for use 
Table 1. The neutron cross section standards.

\begin{tabular}{ll}
\hline Reaction & Recommended Energy Range \\
\hline $\mathrm{H}(\mathrm{n}, \mathrm{n})$ & $1 \mathrm{keV}$ to $20 \mathrm{MeV}$ \\
${ }^{3} \mathrm{He}(\mathrm{n}, \mathrm{p})$ & $0.0253 \mathrm{eV}$ to $50 \mathrm{keV}$ \\
${ }^{6} \mathrm{Li}(\mathrm{n}, \mathrm{t})$ & $0.0253 \mathrm{eV}$ to $1 \mathrm{MeV}$ \\
${ }^{10} \mathrm{~B}(\mathrm{n}, \alpha)$ & $0.0253 \mathrm{eV}$ to $1 \mathrm{MeV}$ \\
${ }^{10} \mathrm{~B}\left(\mathrm{n}, \alpha_{1} \gamma\right)$ & $0.0253 \mathrm{eV}$ to $1 \mathrm{MeV}$ \\
$\mathrm{C}(\mathrm{n}, \mathrm{n})$ & $0.0253 \mathrm{eV}$ to $1.8 \mathrm{MeV}$ \\
$\mathrm{Au}(\mathrm{n}, \gamma)$ & $0.0253 \mathrm{eV}, 0.2$ to $2.5 \mathrm{MeV}$ \\
${ }^{235} \mathrm{U}(\mathrm{n}, \mathrm{f})$ & $0.0253 \mathrm{eV}, 0.15$ to $200 \mathrm{MeV}$ \\
${ }^{238} \mathrm{U}(\mathrm{n}, \mathrm{f})$ & threshold to $200 \mathrm{MeV}$ \\
\hline
\end{tabular}

in the cross section libraries of evaluation projects. Extensive documentation [1] of the work done on the evaluation has been completed and has been submitted for publication. The present report is a summary of that document.

\section{Database studies}

A large database of measurements, a significant portion of which were assembled by Poenitz [2] for the ENDF/B-VI standards evaluation, was used for the evaluation. In addition to the data sets introduced after the ENDF/B-VI evaluation and before the initiation of this evaluation, more than 30 data sets have been added to the standards database. Work has been done to understand the experiments and possible problems with them that may cause discrepancies to exist. During the ENDF/B-VI evaluation process, unusual results [3] were observed with the code GMA [4] when correlated discrepant data were used. To remove problems associated with these discrepancies, data greater than three standard deviations away from the output results were down weighted in the ENDF/BVI GMA evaluation. This had the effect of making $\chi^{2}$ per degree of freedom essentially one. For the R-matrix code EDA [5] that was also used in the ENDF/B-VI standards evaluation, its output covariances were modified by the $\chi^{2}$ per degree of freedom value of the fit. This procedure is equivalent to increasing the uncertainty of all the experimental data in the fit, not just the outliers. This procedure was also used for EDA for the present evaluation. It would be better if the sources of the discrepancies could be found, then the evaluation could be done with consistent data sets. This is a very difficult task since there are thousands of data points. To reduce the effect of discrepant data on the GMA analysis and the RAC [6] Rmatrix evaluation, that was also used for the present evaluation, deviations of experimental measurements from the output of the evaluation were compared with the uncertainties on the data for the database of neutron experiments. The outliers were defined as those for which the difference from the evaluated output value was above two standard deviations for a single point or above one standard deviation for a few sequential points. The uncertainty of outliers was increased by adding an additional component to the covariance matrix for each outlying data set. The length of correlation for this additional medium energy range correlation component was evaluated from an analysis of the energy dependence of the discrepancy. This resulted in a much better $\chi^{2}$ per degree of freedom and

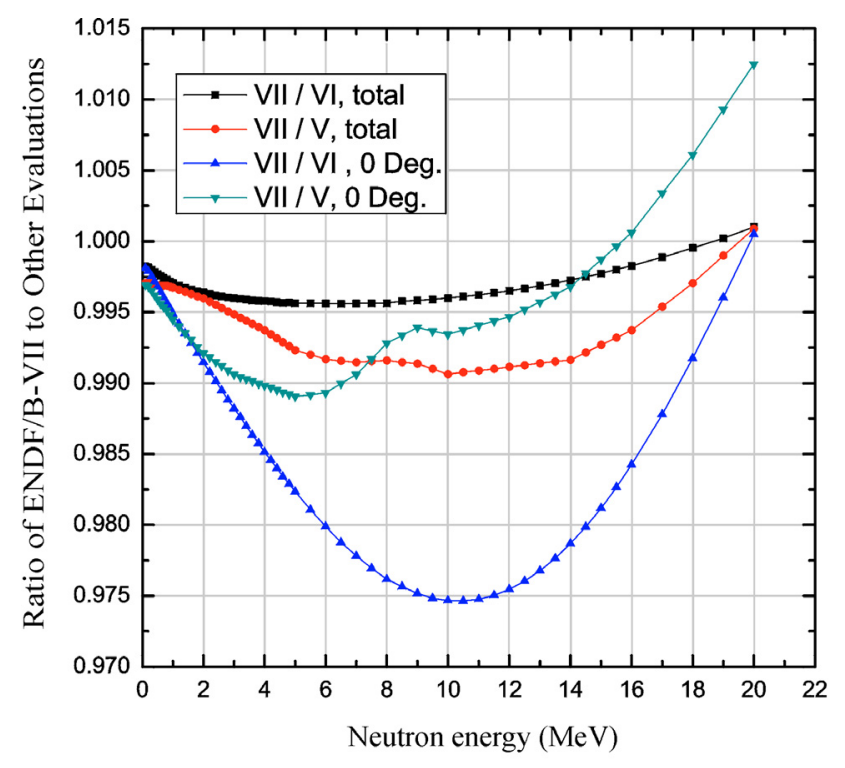

Fig. 1. Comparison of the present hydrogen evaluation to other evaluations. The ENDF/B-VI and ENDF/B-V total cross section are compared with the present results. Also the laboratory zero degree differential cross sections for the ENDF/B-VI and ENDF/B-V evaluations are compared with the results of this evaluation.

larger uncertainty in the evaluated results. The change in the cross section from this procedure was small.

\section{Evaluation of the hydrogen cross section}

An evaluation of the hydrogen scattering cross section below $20 \mathrm{MeV}$ was made at LANL using the R-matrix code EDA. Comparisons of the new hydrogen evaluation with other evaluations are shown in figure 1. With the availability of the new hydrogen standard, all data in the database relative to hydrogen cross sections were converted to the new standard. The database contained measurements relative to several different evaluations of the total cross section and the differential cross section for hydrogen. After this conversion was completed, a hydrogen reevaluation was recommended as a result of concerns about the thermal capture cross section. The reevaluation resulted in small changes in the hydrogen standard cross section. The conversion process was not redone but uncertainties on the converted data were increased to account for the changes in the hydrogen cross section. The effect of the change in the hydrogen standard cross section causes, for example, a change as large as $0.5 \%$ for the evaluated ${ }^{235} \mathrm{U}(\mathrm{n}, \mathrm{f})$ cross section.

\section{Peelle's Pertinent Puzzle}

Early in the evaluation process [7], a test run using a model independent least squares code fitting the logarithm of the cross section produced higher cross sections than a run fitting the cross section. There were discrepant data in the test run. The problem was largely a result of using discrepant data but 
it also was caused by the existence of data correlations. This is the maxi-PPP vs mini-PPP effect. The EDA R-matrix analysis uses only statistical uncertainties for the cross sections, but also includes a procedure for fitting normalizations that takes into account the normalization uncertainties; this procedure does not suffer the PPP problem, as it is equivalent to the Propagated-Uncertainty-Method [8]. Analyses using the RAC R-matrix code that includes medium range correlations do suffer this problem to some degree. A number of methods for reducing PPP have been employed such as using percent uncertainties, using a logarithmic transformation or the BoxCox transformation. The GMA code was modified by adding the Chiba-Smith [9] option to handle PPP problems. This option, called GMAP, renormalizes the experimental absolute errors on the assumption that it is the fractional error that actually reflects the accuracy the experimenter has provided. This approach appears to have reduced the effect of PPP significantly. Comparisons using the Chiba-Smith, Box-Cox and logarithmic transformation are in good agreement for a number of test cases. An important outcome of this work is the observation that special care should be exercised to reduce the effect of discrepant data and PPP to improve the quality of any nuclear data evaluation.

\section{Theoretical model calculations}

Since there are relatively few nucleons in the ${ }^{4} \mathrm{He}$ compound nucleus, it was possible to use the Refined Resonating Group Model (RRGM) to obtain information about the ${ }^{3} \mathrm{He}(\mathrm{n}, \mathrm{p})$ cross section. This model allowed realistic nuclear interactions to be used, however it required very large computer resources. Using effective NN potentials allowed heavier nuclei to be studied such as the $\mathrm{A}=7$ case which provided information on the ${ }^{6} \mathrm{Li}(\mathrm{n}, \mathrm{t})$ standard. Using effective potentials allowed the calculations to be done with a personal computer. The work on these two standards progressed well. The calculations produced results that are rather close to those given by R-matrix analyses. Transforming the RRGM results to R-matrix poles provided guidance for initial values in the R-matrix analyses. This work led to improved values of the parameters and more realistic uncertainties in the cross sections. There were cases where the information on the poles allowed limitations in experimental data sets to be recognized.

\section{The small uncertainty problem}

An important task for the present effort was to try to understand in detail how standard error propagation in model independent or R-matrix analyses could result in very small uncertainties, and whether there were more reasonable corrections or algorithms to employ. The small uncertainties in the ENDF/B-VI standards and the concern about them led to this effort. Work was done on the small uncertainty problem through comparisons of several tests of model independent and R-matrix codes using a common database. The R-matrix codes used in this study were EDA, SAMMY [10], and RAC. The generalized least squares codes used were GLUCS [11], GMAP and SOK [12]. A code based on an analytical approximation model, PADE2 [13] was also used. It was necessary to select a database containing measurements that could be properly used in the comparison. For example, some of the codes could not handle certain types of input data correlations. For the comparison tests, it was assumed that no correlations exist between the data sets. The only correlations within the data sets were assumed to be short energy range (statistical) and long energy range (normalization). The generalized least squares codes were easily found to be in good agreement. Comparisons of the R-matrix codes proved to be more difficult since the input and analysis conditions were difficult to standardize. Studies with SAMMY were particularly useful in understanding differences obtained with different codes, because it was possible to do SAMMY fitting that corresponded essentially to that done by RAC and in a different scheme it was possible to do fitting that corresponded essentially to that by EDA. Good agreement was eventually obtained for the cross sections obtained from the R-matrix analyses. The small differences obtained with the codes could be explained as a result of different procedures, different $\chi^{2}$ expressions and some PPP effects that may be present in the RAC results. The variances and covariances also agreed well with some local differences. A measure of that agreement was given by a comparison of the sum of all elements of the covariance matrix of the evaluated data, which agreed within $1 \%$ for EDA and RAC. These differences were probably mainly due to numerical precisions of the solutions.

The large amount of data for charged-particle-induced reaction channels may be an important factor in the large reduction in the calculated uncertainty. Many charged-particle data, especially differential elastic scattering cross sections, are claimed to have very small uncertainties. It is possible that systematic errors may not have been fully estimated. The option of increasing the uncertainties of outlying data for charged-particle data was used in RAC. These changes and the changes in the neutron database uncertainties noted previously for discrepant data led to somewhat larger uncertainties for the results.

An important result of the present work is that it is essential to consider the covariances, not just the variances, in applications of cross sections to practical systems. Further discussion on the small uncertainties is given in ref. [14].

\section{Evaluation procedure}

A combination procedure similar to that used for the ENDF/B-VI standards evaluation was used to obtain the standards. All the standards except the $\mathrm{H}(\mathrm{n}, \mathrm{n}),{ }^{3} \mathrm{He}(\mathrm{n}, \mathrm{p})$ and $\mathrm{C}(\mathrm{n}, \mathrm{n})$ cross sections were evaluated using the GMAP code to combine experimental data using input from the RAC and EDA R-matrix analyses, and a thermal constants evaluation.

The Axton evaluation [15] of the thermal constants with the associated variance-covariance data for ${ }^{233} \mathrm{U},{ }^{235} \mathrm{U},{ }^{239} \mathrm{Pu}$ and ${ }^{241} \mathrm{Pu}$ was used as input to the GMAP code since it includes accurate cross sections that have been measured relative to the neutron cross section standards. Thus this evaluation would have an impact on the determination of the standards.

Both shape and absolute cross section measurements and their ratios were used as input to GMAP. Also included are 
data involving the ${ }^{238} \mathrm{U}(\mathrm{n}, \gamma)$ and ${ }^{239} \mathrm{Pu}(\mathrm{n}, \mathrm{f})$ cross sections. There are many accurate measurements of these cross sections. The use of these additional data improves the database as a result of ratio measurements of those cross sections to the traditional standards. Measurements of the ${ }^{235} \mathrm{U}$ and ${ }^{239} \mathrm{Pu}$ fission cross sections in the ${ }^{252} \mathrm{Cf}$ spontaneous fission neutron spectrum were also included in the database. These data can be obtained with high accuracy and are only weakly dependent on the uncertainties in the ${ }^{252} \mathrm{Cf}$ spontaneous neutron fission spectrum.

The R-matrix analyses used charged-particle data and the lithium and boron neutron databases, including total and scattering cross section data for these nuclides. The only lithium and boron data for direct use in the GMAP code were the ratio measurements. The R-matrix and GMAP data are totally independent of each other. There are no common data sets and no data sets that have correlations between the R-matrix and GMAP data. For the ${ }^{6} \mathrm{Li}(\mathrm{n}, \mathrm{t}),{ }^{10} \mathrm{~B}(\mathrm{n}, \alpha)$ and ${ }^{10} \mathrm{~B}\left(\mathrm{n}, \alpha_{1} \gamma\right)$ $\mathrm{R}$-matrix work, the cross sections obtained from the RAC and EDA analyses were not identical. The cross sections from the RAC and EDA analyses were averaged (unweighted) and used as the R-matrix input to GMAP. The covariance matrix used with these central values was that from the RAC code since its results appeared more physically reasonable. The R-matrix input and thermal constants data were treated like the additions of other data sets to the GMAP code. At each energy point, half the difference between the RAC and EDA results was treated as a model uncertainty that was added to the RAC covariance matrix. This then takes into account the differences obtained between the RAC and EDA analyses.

\section{Smoothing of the evaluation}

For the ${ }^{6} \mathrm{Li}(\mathrm{n}, \mathrm{t}),{ }^{10} \mathrm{~B}\left(\mathrm{n}, \alpha_{1} \gamma\right)$, and ${ }^{10} \mathrm{~B}(\mathrm{n}, \alpha)$ cross sections smoothing was not required since the highest weight went to the cross sections used in the R-matrix analyses. It was determined that a simple smoothing algorithm would be satisfactory for most cases. It was used sparingly for the heavy element cross sections. A patch using the shape of the Maslov [16] evaluated curve was applied in the $50-60 \mathrm{MeV}$ region for the ${ }^{235} \mathrm{U}(\mathrm{n}, \mathrm{f})$ cross section where a rather large fluctuation, assumed to be statistical, occurred.

\section{Results of the evaluation}

The cross sections obtained from the combining procedure for the ${ }^{6} \mathrm{Li}(\mathrm{n}, \mathrm{t}),{ }^{10} \mathrm{~B}(\mathrm{n}, \alpha),{ }^{10} \mathrm{~B}\left(\mathrm{n}, \alpha_{1} \gamma\right), \mathrm{Au}(\mathrm{n}, \gamma),{ }^{235} \mathrm{U}(\mathrm{n}, \mathrm{f})$, and ${ }^{238} \mathrm{U}(\mathrm{n}, \mathrm{f})$ standard cross sections as well as the ${ }^{238} \mathrm{U}(\mathrm{n}, \gamma)$ and ${ }^{239} \mathrm{Pu}(\mathrm{n}, \mathrm{f})$ cross sections are shown in figures 2-11 compared with the ENDF/B-VI evaluations. For the ${ }^{239} \mathrm{Pu}(\mathrm{n}, \mathrm{f})$ cross section, there are results up to $200 \mathrm{MeV}$, however above $20 \mathrm{MeV}$ comparisons can not be made with the ENDF/B-VI evaluation since it extends only to $20 \mathrm{MeV}$. All uncertainties shown are one standard deviation values for the present evaluation. There is a general increase for most of the standards by as much as 5\% compared with the ENDF/B-VI evaluations. Much of this results from increases in the ${ }^{235} \mathrm{U}(\mathrm{n}, \mathrm{f})$ cross section since so many measurement of standards have been made relative
Table 2. The thermal $(0.0253 \mathrm{eV})$ constants obtained from the standards evaluation with their uncertainties. The values in parenthesis are the values in the ENDF/B-VII file. The constants shown on the rows are fission, capture, elastic scattering, Westcott g-factors for fission and absorption, and total neutrons per fission. The ${ }^{235} \mathrm{U}(\mathrm{n}, \mathrm{f})$ thermal cross section is the only standard in this table.

\begin{tabular}{lllll}
\hline Quantity & ${ }^{233} \mathrm{U}$ & ${ }^{235} \mathrm{U}$ & ${ }^{239} \mathrm{Pu}$ & ${ }^{241} \mathrm{Pu}$ \\
\hline$\sigma_{\text {nf }}(\mathrm{b})$ & 531.22 & 584.33 & 750.00 & 1013.96 \\
& $(531.22)$ & $(585.09)$ & $(747.40)$ & $(1011.85)$ \\
& $\pm 0.25 \%$ & $\pm 0.17 \%$ & $\pm 0.24 \%$ & $\pm 0.65 \%$ \\
\hline$\sigma_{\text {n }}(\mathrm{b})$ & 45.56 & 99.40 & 271.50 & 361.79 \\
& $(45.24)$ & $(98.69)$ & $(270.33)$ & $(363.05)$ \\
& $\pm 1.50 \%$ & $\pm 0.72 \%$ & $\pm 0.79 \%$ & $\pm 1.37 \%$ \\
\hline$\sigma_{\text {nn }}(\mathrm{b})$ & 12.11 & 14.087 & 7.800 & 12.13 \\
& $(12.15)$ & $(15.08)$ & $(7.975)$ & $(11.24)$ \\
& $\pm 5.48 \%$ & $\pm 1.56 \%$ & $\pm 12.30 \%$ & $\pm 21.50 \%$ \\
\hline $\mathrm{g}_{\text {f }}$ & 0.9956 & 0.9773 & 1.0554 & 1.0454 \\
& $(0.9966)$ & $(0.9764)$ & $(1.0542)$ & $(1.046)$ \\
& $\pm 0.14 \%$ & $\pm 0.08 \%$ & $\pm 0.20 \%$ & $\pm 0.53 \%$ \\
\hline $\mathrm{g}_{\mathrm{a}}$ & 0.9996 & 0.9788 & 1.0780 & 1.0440 \\
& $(0.9994)$ & $(0.9785)$ & $(1.0782)$ & $(1.042)$ \\
& $\pm 0.11 \%$ & $\pm 0.08 \%$ & $\pm 0.22 \%$ & $\pm 0.19 \%$ \\
\hline$v$-bar & 2.497 & 2.4355 & 2.8836 & 2.9479 \\
& $(2.497)$ & $(2.4367)$ & $(2.8789)$ & $(2.9453)$ \\
& $\pm 0.14 \%$ & $\pm 0.09 \%$ & $\pm 0.16 \%$ & $\pm 0.18 \%$ \\
\hline
\end{tabular}

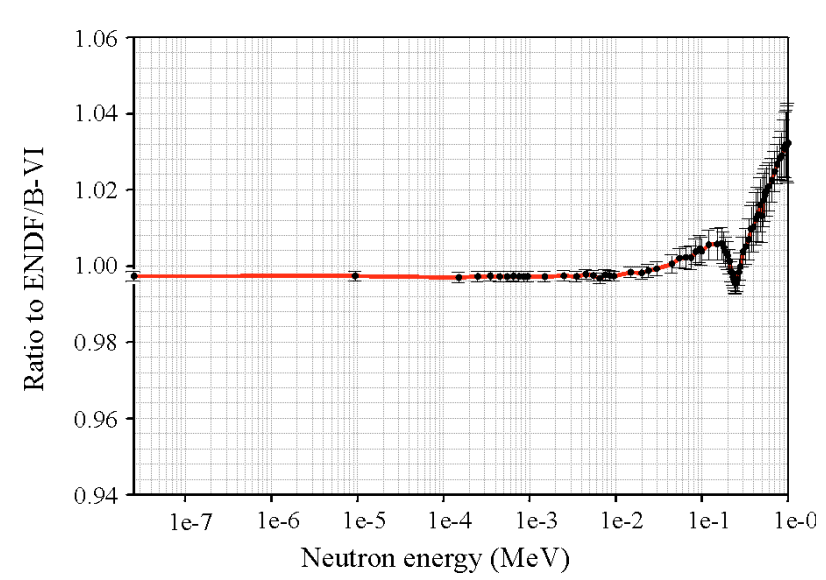

Fig. 2. Comparison of the ${ }^{6} \operatorname{Li}(n, t)$ cross section from this evaluation with the ENDF/B-VI evaluation.

to that cross section. Table 2 shows the thermal constants obtained from this evaluation. Also shown in the table are the values in the ENDF/B-VII neutron sublibrary. The small differences shown in this table between the values obtained from this work and the ones in the neutron sublibrary are because ENDF evaluators have been allowed to make small changes, usually within the experimental uncertainty, in order to optimize the performance in integral tests.

\section{Conclusions and recommendations}

This international effort led to the successful evaluation of the neutron cross section standards that were first used in the new ENDF/B-VII library. It is important to continue to maintain and improve the database and the analysis procedures used 


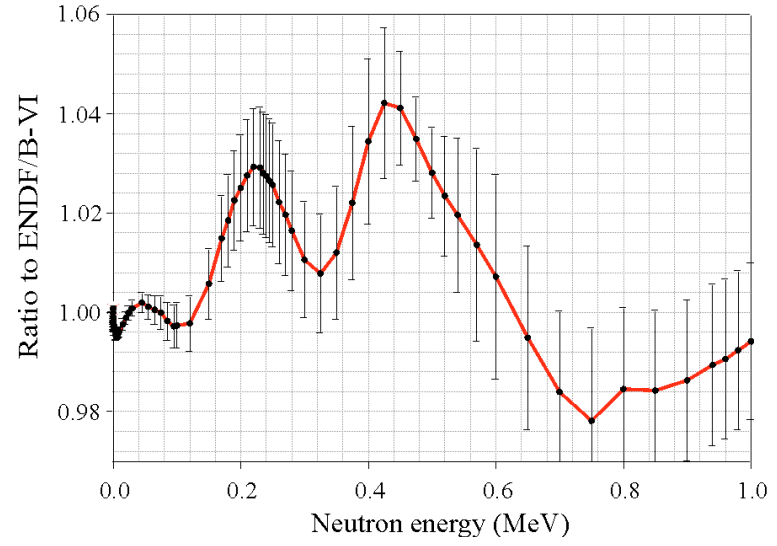

Fig. 3. Comparison of the ${ }^{10} \mathrm{~B}(\mathrm{n}, \alpha)$ cross section from this evaluation with the ENDF/B-VI evaluation.

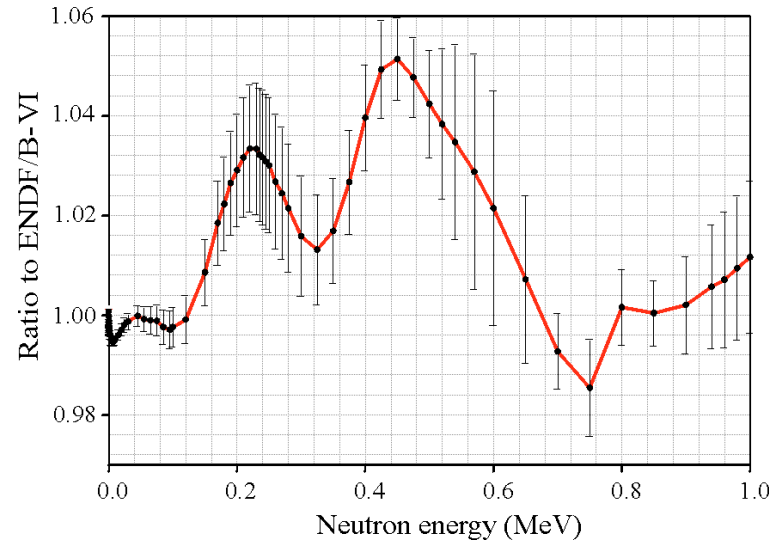

Fig. 4. Comparison of the ${ }^{10} \mathrm{~B}\left(\mathrm{n}, \alpha_{1} \gamma\right)$ cross section from this evaluation with the ENDF/B-VI evaluation.

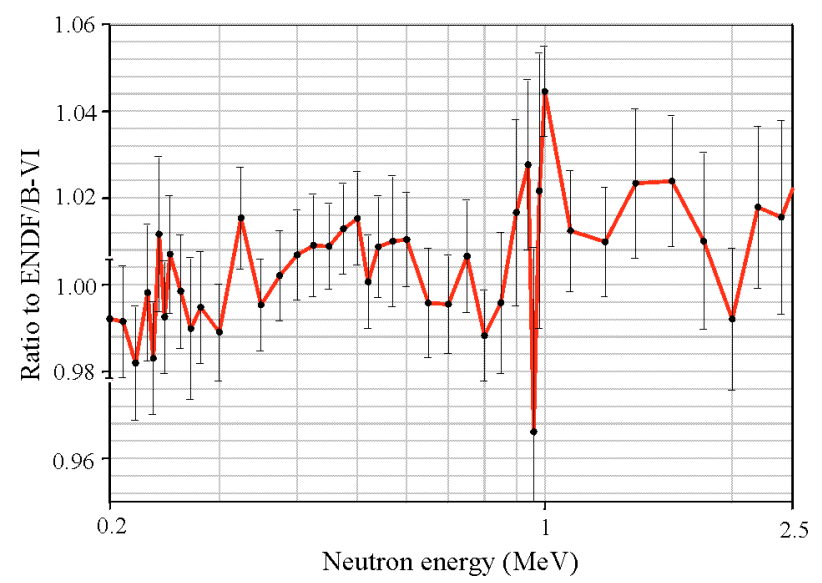

Fig. 5. Comparison of the $\mathrm{Au}(\mathrm{n}, \gamma)$ cross section from this evaluation with the ENDF/B-VI evaluation.

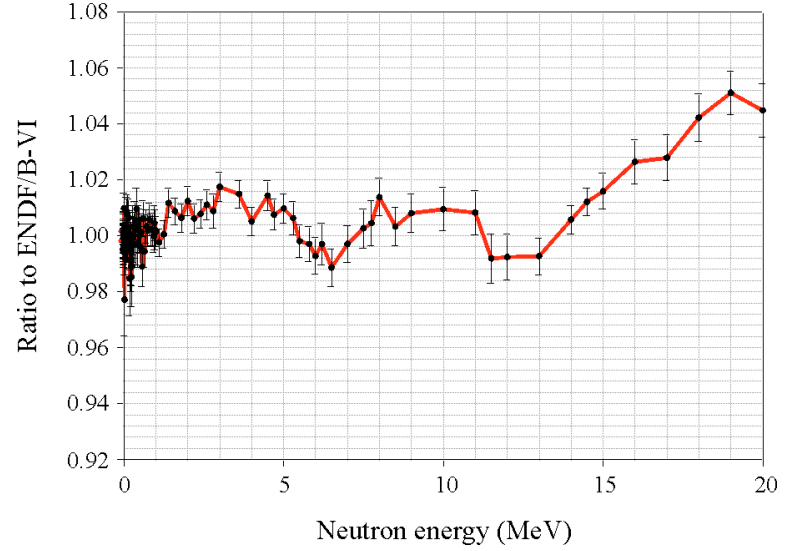

Fig. 6. Comparison of the ${ }^{235} \mathrm{U}(\mathrm{n}, \mathrm{f})$ cross section up to $20 \mathrm{MeV}$ from this evaluation with the ENDF/B-VI evaluation.

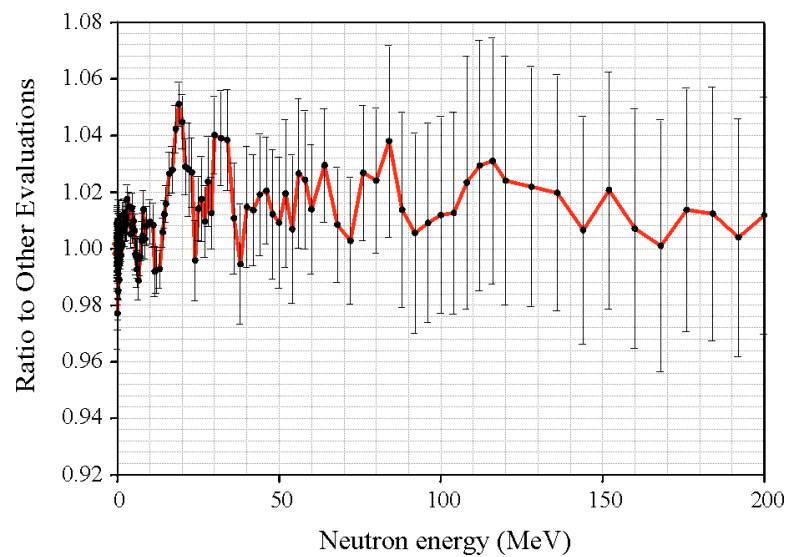

Fig. 7. Comparison of the ${ }^{235} \mathrm{U}(\mathrm{n}, \mathrm{f})$ cross section from this evaluation with the ENDF/B-VI evaluation up to $20 \mathrm{MeV}$ and with updated values [19] above $20 \mathrm{MeV}$.

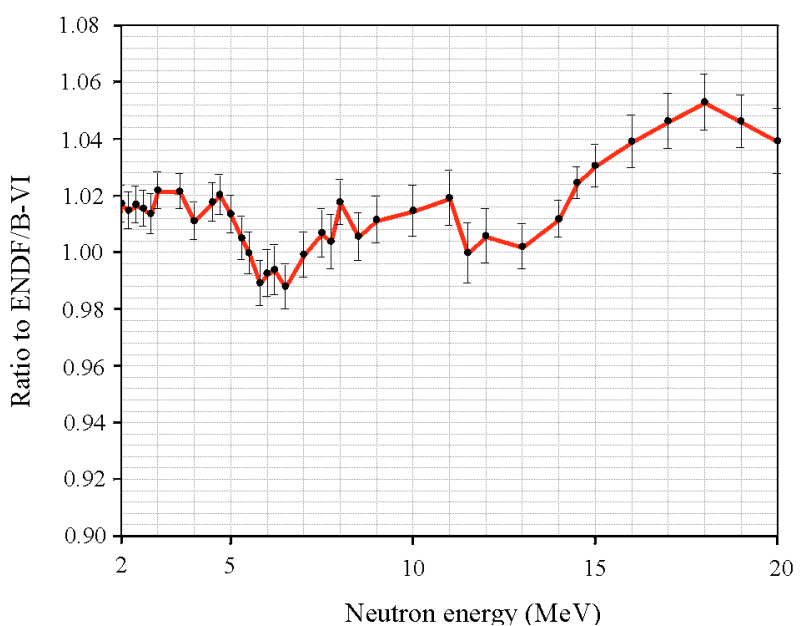

Fig. 8. Comparison of the ${ }^{238} \mathrm{U}(\mathrm{n}, \mathrm{f})$ cross section up to $20 \mathrm{MeV}$ from this evaluation with the ENDF/B-VI evaluation. 


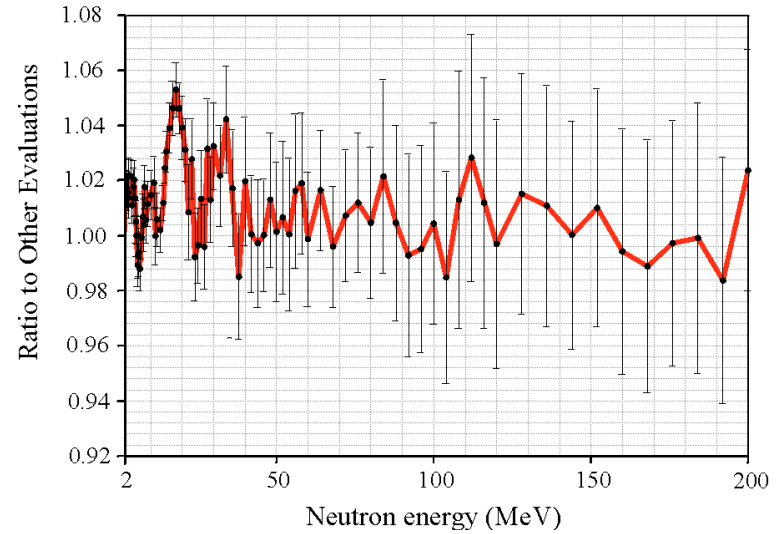

Fig. 9. Comparison of the ${ }^{238} \mathrm{U}(\mathrm{n}, \mathrm{f})$ cross section from this evaluation with the ENDF/B-VI evaluation up to $20 \mathrm{MeV}$ and with updated values [19] above $20 \mathrm{MeV}$.

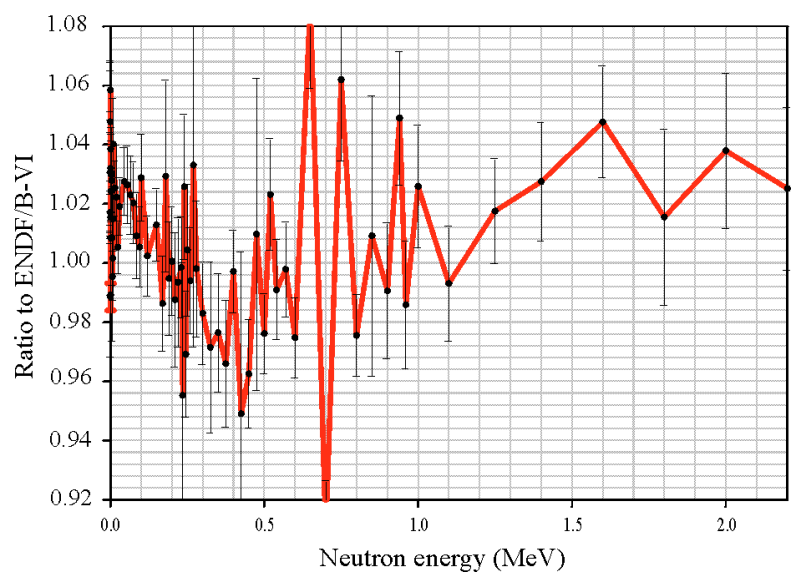

Fig. 10. Comparison of the ${ }^{238} U(n, \gamma)$ cross section from this evaluation with the ENDF/B-VI evaluation.

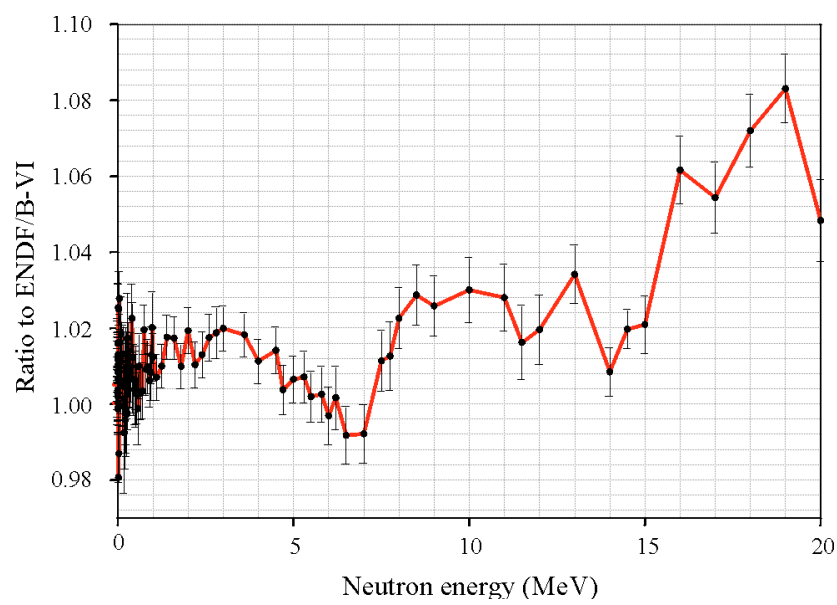

Fig. 11. Comparison of the ${ }^{239} \mathrm{Pu}(\mathrm{n}, \mathrm{f})$ cross section from this evaluation with the ENDF/B-VI evaluation. in determining these standards. More work should be done on physical models for smoothing of the fission and capture standards. A new IAEA Data Development Project has been approved that is focused on the maintenance of the neutron cross section standards. This project should provide a method for obtaining standards evaluations that will be up-to-date when they are needed by the various nuclear data evaluation projects. An important result of the present work is that it is essential to consider the covariances, not just the variances in cross section applications.

The authors wish to thank the CSEWG, the WPEC and the IAEA for their support of this evaluation project.

\section{References}

1. IAEA Technical Series Report (to be published in 2007).

2. W.P. Poenitz, S.E. Aumeier, Argonne National Laboratory Report ANL/NDM-139, 1997.

3. A.D. Carlson et al., Brookhaven National Laboratory Report, ENDF-351, 1993.

4. W.P. Poenitz, Brookhaven National Laboratory Report BNLNCS-51363, Vol. I, 1981, pp. 249-289.

5. G.M. Hale, Brookhaven National Laboratory Report BNL-NCS51363, Vol. II, 1981, pp. 509-531.

6. Z.P. Chen, At. Energy Sci. Technol. 29, No. 4, 366 (1995) .

7. A.D. Carlson, G.M. Hale, V.G. Pronyaev, IAEA Report INDC (NDS)-438, 2003.

8. N.M. Larson, in Proc. Int. Conf. on Nuclear Data for Sci. and Technology, Santa Fe, 2005, edited by R.G. Haight et al., AIP Conf. Proc. 769, 374 (2005).

9. S. Chiba, D.L. Smith, Argonne National Laboratory Report ANL/NDM-121,1991.

10. N.M. Larson, Oak Ridge National Laboratory Report ORNL/ TM-9179/R7 and ENDF-364/R1, 2006. Available on http://www. ornl.gov/sci/nuclear_science_technology/nuclear_data/sammy/.

11. S. Tagesen, D.M. Hetrick, Proc. of International Conf. on Nuclear Data for Sci. and Technology, Gatlinburg, 1994, edited by J.K. Dickens, Am. Nucl. Soc., Vol. 1 (Inc., LaGrange Park, IL, USA, 1994), pp. 589-591.

12. T. Kawano, et al., JAERI Report JAERI- Research-2000-004, 2000.

13. S.A. Badikov et al., Proc. of International Conference, on Nuclear Data for Science and Technology, Júlich, 1991, edited by S.M. Qaim (Springer-Verlag, 1991), pp. 182-187.

14. E.V. Gai, V.G. Pronyaev (these proceedings).

15. E.J. Axton, CBNM (IRMM) Report GE/PH/01/86, 1986.

16. V.M. Maslov, Eur. Phys. J. A21, 281 (2004).

17. J. Hardy (private communication), Memo dated 18 June 1985.

18. R.E. MacFarlane (2006) (private communication).

19. A.D. Carlson, et al., IAEA Report INDC(NDS)-368, 1997. 